\title{
Elaboración de Membranas Poliméricas Porosas a partir de Cristales Coloidales
}

\author{
Natalia Casis ${ }^{1,2}$, Maria M. Fidalgo ${ }^{2}$, Serge Ravaine ${ }^{3}$ y Diana A. Estenoz ${ }^{1}$ \\ (1) INTEC (Universidad Nacional del Litoral - CONICET), Guemes 3450, Santa Fe-Argentina. \\ (2) Departamento de Ingeniería Química, ITBA, Madero 399, Buenos Aires-Argentina. \\ (3)Centre de Recherche Paul Pascal, Avenue Albert Schweitzer, F-33600 Pessac-Francia \\ (e-mail: ncasis@santafe-conicet.gov.ar)
}

\begin{abstract}
Resumen
Se elaboraron membranas poliméricas porosas con elevado control de la morfología y de los tamaños de poros a partir de cristales coloidales constituidos por partículas de sílice. El método empleado permite obtener diferentes morfologías dependiendo del tamaño de las partículas y del número de capas involucradas en los depósitos. Para la síntesis de las partículas se emplearon dos métodos, originándose distintos tamaños. Los cristales coloidales se obtuvieron por deposición vertical de las partículas sobre un sustrato de vidrio y por la técnica de Langmuir-Blodgett. Se fabricaron membranas de poliestireno (PS) y de copolímero de estireno (St)-vinil bencil timina (VBT). El procedimiento involucró la infiltración de los monómeros seguida por la polimerización por irradiación con luz UV. El método propuesto provee una alternativa relativamente simple, muy versátil y a la vez económica para la elaboración de polímeros macroporosos.
\end{abstract}

Palabras clave: membranas poliméricas, poliestireno, sílice, cristales coloidales, tamaños de poro

\section{Elaboration of Porous Polymeric Membranes from Colloidal Crystals}

\begin{abstract}
Polymeric porous membranes with a high control of morphology and porous size were fabricated from colloidal crystals made of silica particles. This fabrication process allows different morphologies according to the particle sizes and number of layers on the templates. Silica particles with a narrow size distribution were synthesized by two methods. Colloidal crystals were obtained by vertical deposition of the particles on a glass substrate and by the Langmuir-Blodgett technique to create templates of different particle sizes. To produce the membrane, polystyrene (PS) and styrene (St)vinyl benzyl thymine (VBT) copolymers were used. The procedure involved the infiltration of monomers in the templates and then, the polymerization by UV radiation. The proposed method represents a relatively simple alternative, both versatile and economically attractive to manufacture macroporous polymers.
\end{abstract}

Keywords: polymeric membranes, polystyrene, silica, colloidal crystals, porous size 


\section{INTRODUCCIÓN}

La selectividad y la eficiencia de los procesos de separación por membranas porosas están determinadas por propiedades tales como porosidad, distribución de tamaños de poro, carga superficial, ángulo de contacto, etc. Esto implica que tanto la morfología de la estructura porosa como el material que la compone cumplen roles determinantes en las características del proceso de filtración por membranas (Mulder, 2004).

Las membranas se clasifican de acuerdo al tamaño de sus poros: Nanofiltración: $\leq 3 \mathrm{~nm}$; Ultrafiltración: 10-100 nm y Microfiltración: <1 $1 \mu \mathrm{m}$. Los sistemas de ultrafiltración y microfiltración se emplean para la remoción de material particulado y coloidal en tratamientos de aguas salobres. Los materiales más utilizados son polímeros sintéticos tales como polisulfona, polietersulfona, poliacrilonitrilo, ésteres de celulosa, polimidas, poliamidas alifáticas, y polietercetona (Mulder, 2004). Estas membranas presentan elevados costos, tazas de flujo pequeñas y tiempo de vida útil limitado. Actualmente se están desarrollando nuevos materiales poliméricos por modificación en la estructura de aquellos polímeros convencionales tendiendo a incrementar el flujo de agua a través de la membrana a presiones reducidas y mejorando la retención del material disuelto o suspendido para prolongar así, su tiempo de vida y aumentar la selectividad (Vainrot et al., 2008; Tang et al., 2009).

Por otra parte, los métodos de fabricación de estas membranas porosas en aplicación comercial no alcanzan a conseguir un alto control en la distribución del tamaño de poros obtenidos ni de la morfología, además de estar relacionados a materiales específicos (Mulder, 2004). Es deseable obtener nuevos métodos de fabricación de membranas que permitan el diseño de la estructura a la nanoescala, siendo estos adaptables a una amplia variedad de materiales.

Se pueden crear sólidos porosos por deposición de nanopartículas esféricas formando un cristal coloidal (Jiang et al., 1999a; Velev et al., 1997, Johnson et al., 1999). Si se cambian las condiciones de deposición de las partículas, se obtienen diferentes tipos de estructuras de los cristales y por lo tanto se obtendrán distintas morfologías de la membrana. La estructura puede ser altamente organizada o totalmente asimétrica, con capas de diferentes tamaños de poro. Estas últimas son muy comunes en los procesos de filtración, donde el soporte se compone de poros más grandes que le confieren a la membrana fuerza mecánica, y una capa activa más fina constituida por poros más pequeños que determina las propiedades de selectividad y permeabilidad. El soporte permite reducir el espesor de la capa fina aumentando la permeabilidad de la membrana. Este tipo de estructura compuesta se logra en el proceso de obtención de los cristales coloidales, al depositar capas de diferentes tamaños de partículas una sobre la otra siguiendo la técnica de Langmuir-Blodgett (Reculusa y Ravaine, 2003; Reculusa et al., 2004; Van Duffel et al., 2001; Cortalezzi et al., 2005).

Este trabajo propone la fabricación de membranas poliméricas porosas de poliestireno (PS) y copolímero de estireno (St)-vinil bencil timina (VBT) a partir de los depósitos de partículas de $\mathrm{SiO}_{2}$. El monómero VBT es similar al St y ha sido desarrollado de manera de incorporar la fotorreactividad de un grupo timina en el sistema polimérico (Fig. 1). Los copolímeros basados en la vinil-bencil-timina (VBT) constituyen una nueva familia de materiales que se entrecruzan cuando son irradiados con luz UV ( $254 \mathrm{~nm})$ (Grasshoff et al. 1995, Anastas y Warner, 1998). El entrecruzamiento modifica algunas propiedades del material con respecto al homopolímero de PS por ejemplo mejora su resistencia mecánica con lo cual en el caso de las membranas, le permitiría soportar mayores presiones en el proceso de filtrado. La elasticidad, solubilidad y fluencia del material dependerán del grado de entrecruzamiento alcanzado.

Los cristales coloidales se logran por deposición de las partículas de $\mathrm{SiO}_{2}$ sobre placas de vidrio. Mediante la modificación de los parámetros de obtención de estos depósitos se logra variar la morfología del film a obtener, mientras que el tamaño de los poros estará relacionado con el tamaño de las partículas de $\mathrm{SiO}_{2}$ empleadas (Jiang et al., 1999a; 1999b). Estas estructuras con alto grado de control de la morfología y de la porosidad tienen una potencial aplicación en procesos en los que se requiera un excelente nivel de separación con alta selectividad. Asimismo, abren alternativas interesantes para la fabricación de sensores medioambientales dado su alta área específica y la variedad de materiales en los que pueden obtenerse los films. 


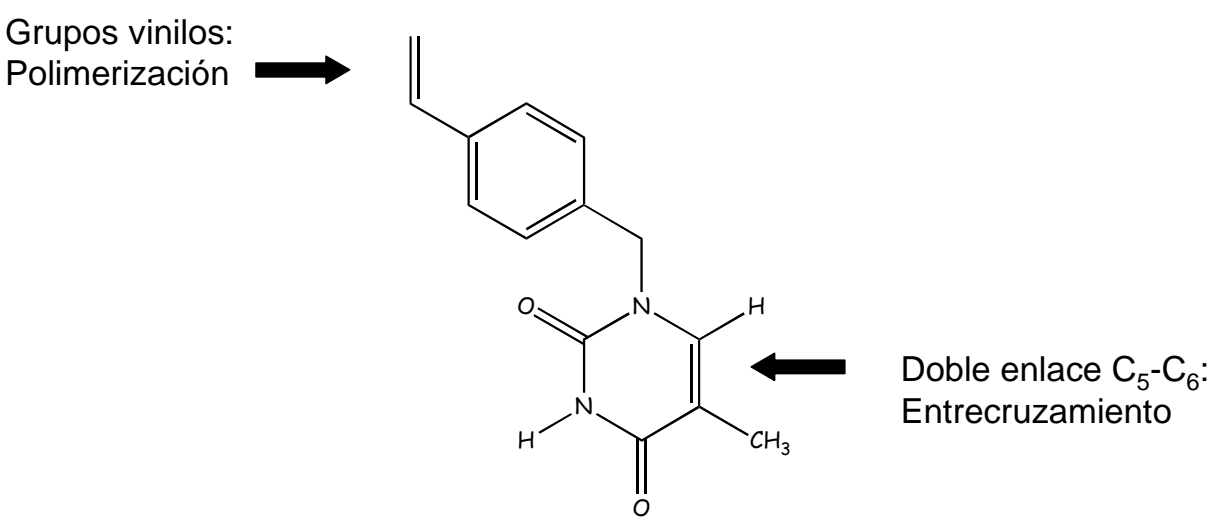

Fig. 1: Reactividad del VBT

\section{MATERIAL Y MÉTODOS}

Materiales: se empleó etanol (J.T. Baker), solución de amonio (30\% en agua, Merck), tetraetoxisilano (TEOS) (99\%, Fluka), alil trimetoxisilano (ABCR), ácido fluorhídrico 49\% (Fisher). Estos reactivos se obtuvieron bajo calidad analítica y se utilizaron sin previas purificaciones. El monómero de St (grado técnico, Petrobras Energía, S.A.) fue previamente destilado con vacío. El VBT se sintetizó a partir de la timina y el cloruro de vinil bencilo (Grasshoff et al., 1995).

El método de obtención de las membranas porosas consta de las siguientes etapas: síntesis de las nanopartículas, preparación de los cristales coloidales y fabricación de las membranas macroporosas, las cuales se detallan a continuación.

\section{Síntesis de las Nanopartículas de $\mathrm{SiO}_{2}$}

Las nanopartículas de $\mathrm{SiO}_{2}$ se sintetizaron por dos métodos diferentes: 1) Stober-Fink-Bohn (Stober et al., 1968) para obtener partículas de diámetro medio $\leq 300 \mathrm{~nm}$ y 2) Kang (Kang et al., 2001) para partículas de diámetro medio $\geq 600 \mathrm{~nm}$.

Método uno, el TEOS se adicionó al etanol y a la solución de amonio. La mezcla resultante se agitó aproximadamente durante $12 \mathrm{~h}$ hasta finalizar la reacción. Método dos, el TEOS disuelto en $40 \mathrm{~mL}$ de etanol se adicionó a la solución de amonio y etanol remanente a una velocidad de $8 \mathrm{ml} / \mathrm{h}$ mediante una jeringa conectada a una bomba. Los métodos y las condiciones experimentales empleadas se presentan en la Tabla 1.

Tabla 1: Métodos y Condiciones Experimentales

\begin{tabular}{lcc}
\hline & Stober-Fink-Bohn & Kang \\
\hline TEOS & $15 \mathrm{ml}$ & $10 \mathrm{ml}$ \\
Etanol & $400 \mathrm{ml}$ & $140 \mathrm{ml}$ \\
Solución de Amonio (30\%) & $37.5 \mathrm{ml}$ & $22 \mathrm{ml}$ \\
Velocidad de agitación & $300 \mathrm{rpm}$ & $300 \mathrm{rpm}$ \\
Tiempo & $12 \mathrm{~h}$ & $6 \mathrm{~h}$ \\
Temperatura & $25^{\circ} \mathrm{C}$ & $25^{\circ} \mathrm{C}$ \\
\hline
\end{tabular}

\section{Preparación de los Cristales Coloidales}

Las superficies de las partículas fueron funcionalizadas con alil trimetoxisilano. El objetivo de esta etapa fue evitar la agregación de las mismas cuando se dispersan sobre la interface agua-aire en la cuba de Langmuir. Las cantidades adicionadas por $\mathrm{ml}$ de suspensión de partículas fueron: $7 \times 10^{-4} \mathrm{~g}$ para el método uno y $2 \times 10^{-4} \mathrm{~g}$ para el método dos, estos cálculos se realizaron teniendo en cuenta la concentración y el área superficial de las partículas (Reculusa y Ravaine, 2003). El sistema se agitó a 
temperatura ambiente por $1 \mathrm{~h}$ y luego se calentó hasta $\mathrm{T}=90^{\circ} \mathrm{C}$, una vez alcanzada la temperatura se continuó la reacción por 2 hs más.

Los depósitos de partículas de $\mathrm{SiO}_{2}$ se prepararon mediante dos técnicas: a) autoensamblado (único tamaño de partículas) (Denkov et al., 1993) y b) deposición capa por capa tipo Langmuir-Blodgett (2 tamaños de partículas) (Reculusa y Ravaine, 2003).

En la técnica por autoensamblado, la placa de vidrio que sirvió de soporte se colocó dentro de un vaso que contenía la suspensión de las partículas en etanol. Se dejó evaporar el alcohol a temperatura ambiente (aproximadamente 2 a 3 días) obteniéndose así los depósitos.

En la deposición capa por capa, las partículas se colocaron dentro de una cuba de Langmuir. Una vez en la cuba, las partículas se dispersaron sobre la interface aire-agua y se comprimieron mediante barreras móviles para luego ser transferidas una capa por vez sobre el sustrato sólido de vidrio.

Fabricación de las Membranas Macroporosas

Se fabricaron membranas macroporosas de PS puro y de copolímero de St-VBT. El depósito con las partículas de $\mathrm{SiO}_{2}$ se cubrió con una segunda placa de vidrio formando un sándwich y se lo puso en contacto con el St ó con la solución de St-VBT (1\% p/p) produciéndose la infiltración por capilaridad (Fig. 2a). La polimerización se realizó por exposición a la luz UV a $\lambda=360 \mathrm{~nm}$ por $72 \mathrm{hs}$. Para el caso de St-VBT, se realizó luego una segunda exposición a $\lambda=254 \mathrm{~nm}$ por $30 \mathrm{~min}$. para producir el entrecruzamiento de los grupos timina (Fig. 2b). El film polimérico se puso en contacto con una solución acuosa de ácido fluorhídrico $49 \%$ diluida con agua ultrapura al 5\%, que por ataque al vidrio permitió la separación del mismo y la disolución de las partículas de $\mathrm{SiO}_{2}$, dejando una estructura porosa que fue el "negativo" del depósito original.

Las partículas y las membranas poliméricas se observaron mediante un microscopio electrónico de barrido (SEM) modelo JEOL JSM-840A.

a)

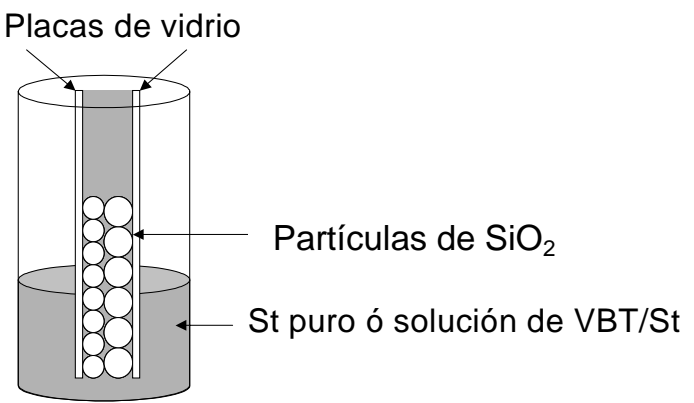

b)

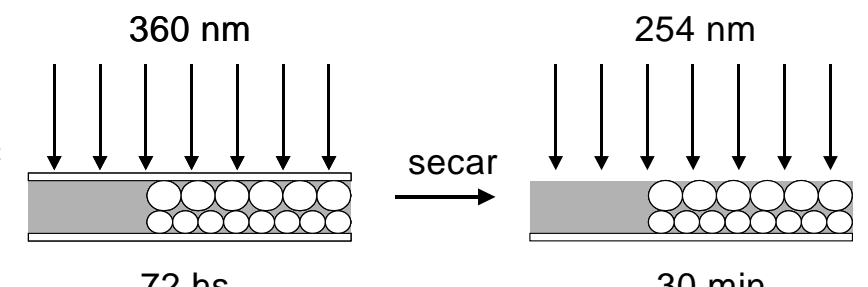

72 hs
$30 \mathrm{~min}$.

Fig. 2: Esquema del procedimiento de obtención de las membranas. a) Infiltración con St ó con solución VBT-St. b) Irradiación de los depósitos.

\section{RESULTADOS Y DISCUSIÓN}

Se obtuvieron partículas monodispersas cuyos diámetros medios fueron: $290 \mathrm{~nm}$ con una desviación standard relativa de $2.57 \%$ para el método uno, y $1090 \mathrm{~nm}, 4.76 \%$ para el método dos (Fig. 3). Como se aprecia en la figura las partículas tienden a agruparse en arreglos hexagonales y este es un buen parámetro de monodispersidad.

Las Figs. 4 y 5 muestran las micrografías de las membranas poliméricas obtenidas. Las Figs $4 a$ ) y 5a) muestran la porosidad superficial (vista superior de la membrana). En las Figs. 4b) y $5 b$ se observa la porosidad interna. Las cavidades que constituyen la porosidad de la membrana tienen aproximadamente las mismas dimensiones que las partículas utilizadas, 290 y $1090 \mathrm{~nm}$. El tamaño de poro efectivo de la membrana está dado por las conexiones entre las cavidades que han dejado las partículas y estas interconexiones dependerán tanto del tamaño de la partícula como de la viscosidad del monómero o solución de monómeros utilizados. Para determinar el tamaño de poro 
efectivo más de 100 poros fueron contabilizados y medidos en SEM obteniéndose así, su valor medio y desviación estándar. En el caso de la membrana obtenida a partir de St el tamaño de poro efectivo obtenido para las partículas de $1090 \mathrm{~nm}$ fue de $135.6 \pm 7.3 \mathrm{~nm}$ [Fig. 4a)] y en el caso de la obtenida a partir de St-VBT (solución más viscosa) el tamaño de poro efectivo fue de $200.3 \pm 9.7 \mathrm{~nm}$ [Fig. 5a)]. Para las partículas más pequeñas $(290 \mathrm{~nm})$, el tamaño de poro efectivo obtenido para la membrana de PS fue de $60.8 \pm 2.3 \mathrm{~nm}$ y para la de St-VBT fue de $92.3 \pm 3.7 \mathrm{~nm}$. Esto se debe al aumento de la viscosidad de la solución de monómeros (St-VBT) respecto al St puro, las partículas no alcanzan a cubrirse completamente y se obtienen poros de mayor tamaño.

La técnica de Langmuir Blodgett para la elaboración de los cristales coloidales permitió un mejor control del espesor de las membranas relacionado con el tamaño partículas y el número de capas o deposiciones involucrados. Además, la superposición de capas de partículas de diferentes tamaños permitió obtener estructuras asimétricas. La Figura 4c) muestra un corte transversal de la membrana asimétrica donde se aprecian las diferentes capas que dejaron los depósitos de partículas de diferentes tamaños.
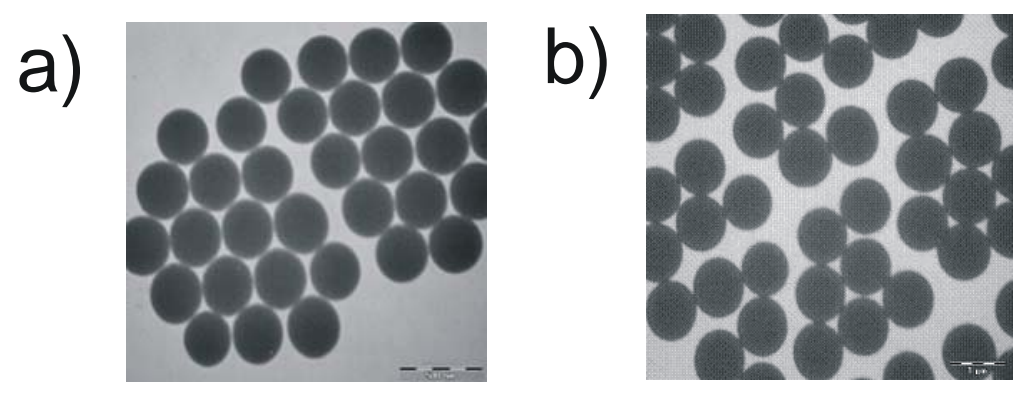

Fig. 3: Micrografías de las partículas de $\mathrm{SiO}_{2}$ obtenidas por SEM. a) 290 nm. b) 1090 nm.

a)

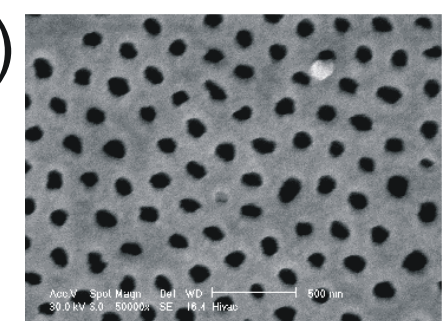

Fig. 4: Membrana porosa de PS. a) b)

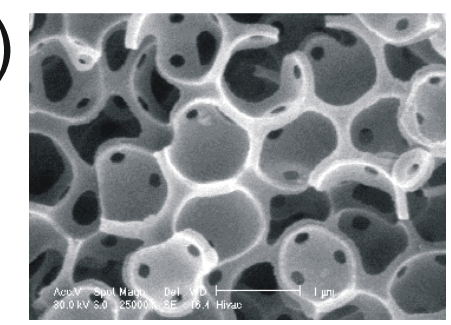

c)

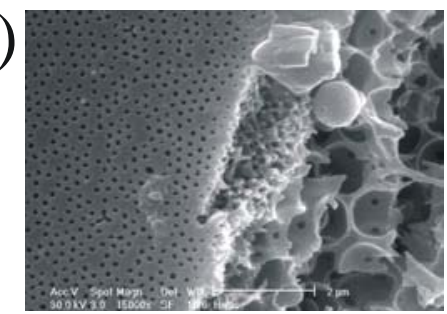

a) Porosidad superficial. b) Porosidad interna. c) Corte transversal de la membrana.

a)

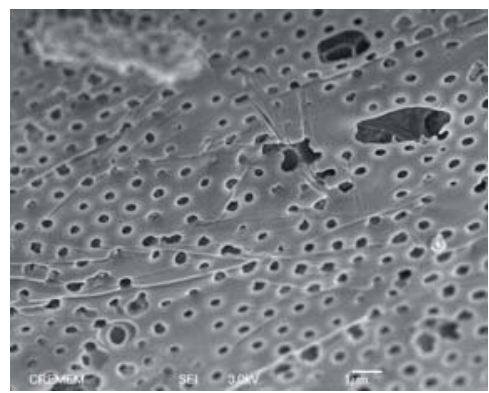

b)

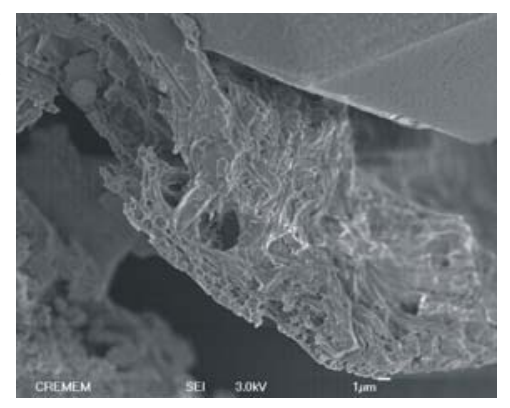

Fig. 5: Membrana porosa de copolímero St-VBT. a) Porosidad superficial. b) Porosidad interna.

\section{CONCLUSIONES}

En cada material sintetizado, el tamaño de los poros tanto en la parte superficial como la parte interna de la membrana quedó determinado por el diámetro de las partículas esféricas que formaban los cristales coloidales. Además, para iguales tamaños de partículas, los diferentes polímeros empleados permitieron obtener membranas con diferentes tamaños de poro relacionados con la viscosidad de los monómeros de partida. 
Mediante este método de elaboración es posible obtener membranas porosas con una amplia variedad de materiales y de tamaños de poros empleando distintos monómeros y utilizando diferentes diámetros de partículas en un rango comprendido entre $100 \mathrm{~nm}$ y $1.5 \mu \mathrm{m}$.

Este método provee una alternativa relativamente simple, muy versátil y a la vez económica para la elaboración de polímeros macroporosos.

En trabajos futuros se pretende evaluar las membranas para ser utilizadas como filtros en el tratamiento de aguas, determinar su permeabilidad, selectividad y optimizar su proceso de obtención para producir membranas con propiedades predeterminadas para aplicaciones específicas.

\section{REFERENCIAS}

Anastas, P.y J. Warner; Green Chemistry: Theory and Practice, NY: Oxford Univ. Press Inc. (1998).

Cortalezzi, M.M., V.L. Colvin y M.R. Wiesner; Controlling Submicron-Particle Template Morphology: Effect of Solvent Chemistry, J. of Colloidal Interface Sci.: 283, 366-372 (2005).

Denkov, N. D. y otros cinco autores; Two-Dimensional Crystallization, Nature: 361, 26 (1993).

Grasshoff, J., L. Taylor y J. Warner; Vinylbenzyl Thymine Monomers. U.S. Patent 5, 455, 349 (1995).

Jiang, P., J. F. Bertone, K. S. Hwang y V. L. Colvin; Single-Crystal Colloidal Multilayers of Controlled Thickness, J. Am. Chem. Soc.: 11, 2132-2140 (1999a).

Jiang, P. y otros cuatro autores; Template-Directed Preparation of Macroporous Polymers with Oriented and Crystalline Arrays of Voids, J. Am. Chem. Soc.: 121, 11630-11637 (1999b).

Johnston, A., P.J. Ollivier y T.E. Mallouk; Ordered Mesoporous Polymers of Tunable Pore Size from Colloidal Silica Templates, Science: 283, 963-965 (1999).

Kang, S. y otros cinco autores; Preparation and Characterization of Epoxy Composites Filled with Functionalized Nanosilica Particles Obtained via Sol-Gel Process, Polymer: 42, 879-887 (2001).

Mulder, M.; Basic Principles of Membrane Technology, 2nd ed., Kluwer Academic Publishers, Dordrecht (2004).

Reculusa, S. y S. Ravaine; Synthesis of Colloidal Crystals of Controllable Thickness through the Langmuir-Blodget Technique, Chem. Mater.: 15, 598-605 (2003).

Reculusa, S., P. Masse y S. Ravaine; Three-Dimensional Colloidal Crystals with a well Defined Architecture, J. of Colloidal Interface, Sci.: 279, 471-478 (2004).

Stober, W., A. Fink y E. Bohn; Controlled Growth of Monodispersed Silica Spheres in the Micron Size. J. of Colloidal Interface, Sci.: 26, 62-69 (1968).

Tang, Z. y otros diez autores; UV-Cured Poly(Vinyl Alcohol) Ultrafiltrtion Nanofibrous Membrane Based on Electrospun Nanofiber scaffolds, J. of Membrane Sci.: 328, 1-5 (2009).

Vainrot, N., M.S. Eisen y R. Semita; Membranes in Desalination and Water Treatment, MRS Bulletin: 33, 16-20 (2008).

Van Duffel, B., R.H.A. Ras, F.C. De Shryver y R.A. Schoonheydt; Langmuir-Blodgett Deposition and Optical Diffraction of two-Dimensional Opals, J. Mater. Chem.: 11, 3333-3336 (2001).

Velev, O.D., T.A. Jede, R.F. Lobo y A.M. Lenhoff; Porous Silica Via Colloidal Crystallization, Nature: 389, 447 (1997). 